\title{
Machiavellianism and Personality Typing as Determinants for Screening Commercial Pilot Candidates
}

\author{
Raymond Allen Hamilton II \\ Auburn University
}

\begin{abstract}
The association between personality and effective team leadership in the cockpit environment has been a major focus of research in aviation management. The major air carriers incorporate in their assessment process personality style and decision-making skills by placing pilot candidates through a two or three stage interview process. In an ongoing effort to develop a comprehensive pilot candidate selection model, university researchers have studied the relationship between personality type and skill in small group manipulation. In this study, the Myers-Briggs Type Indicator as a construct of personality type and the Mach V scale as a construct of skill in small group manipulation were employed in the survey of 52 commercial flight officers. Eight-five percent of the surveyed pilots fell into one of the sixteen personality types measured by the MBTI scale, which correlated significantly with Machiavellian orientation as measured by the Mach V scale. Research indicates a significant relationship in success in leadership of small groups and the Mach V scores. The results of this study suggest the potential of select dimensions of the MBTI and the Mach V instruments in the screening of commercial pilot candidates for hire.
\end{abstract}

\section{INTRODUCTION}

Commercial aviation, in particular the major air carriers, are well aware of the criticality in selecting those pilot candidates with the highest overall return on the training investment. It is expensive, and to fail means not only increased training costs and lost revenue, but the consequences of greater risk of accidents and the resultant fallout of public perception are unacceptable.

It is not enough to 'screen-out' those who fail to meet the needed set of technical qualifications and operational experience. The industry recognizes the need to incorporate into the selection protocol an assessment of personality factors to include attitudes related to crew coordination and effective team work under stressful safety of flight conditions (Chidester et al, 1991). Research suggests that pilot selection protocols may have low predictive validity and their content has remained relatively unchanged over the decades. These protocols may reflect higher predictive validity in the training regimen rather than in that of the operation line (Damos, 1996).

Yet the critical point of screening those who are not amenable to team-oriented training occurs before-not after the applicant becomes an employee. When considering the implicit costs of bringing on board pilots who resist development of team skills, the industry would benefit greatly from a low-cost 'selecting-in' of candidates using a model based on the attitudes and temperament of those pilots having the leadership potential sought.

\section{RESEARCH QUESTIONS}

Given the criticism of current pilot selection protocols, little research has been undertaken to improve them prior to the decision to interview and administer the typical phased selection process. This research aims at providing the first step to screening candidates based on a model reflecting the personality and leadership temperament of those pilots the carrier identifies as optimum. To explore improvements in the initial screening of applicants for interview, the following research questions were raised.

Is there an identifiable personality and leadership temperament associated with those pilots a carrier deems most successful in crew coordination and performance under stress? Given such a temperament, are there instruments 
with the discriminatory power to 'select-in' applicants who meet the personality and leadership temperament profile of the select pilot group?

\section{BACKGROUND}

The Myers-Briggs Type Indicator (MBTI) was chosen as the instrument to evaluate personality temperament. To augment the MBTI in measuring strength of leadership of informal, small groups, Christie and Geis' (1970) Mach V scale was selected. The purpose in this study was to assess the MBTI as a psychological instrument in facilitating the screening of pilot applicants through the development of a discriminate function or variant that would be both reliable and consistent. The Kiersey version of the MBTI was employed (Kiersey \& Bates, 1998). Included as well was the Mach V instrument because of its strong correlation to effective leadership in informal, small groups (Christie \& Geis, 1970). A two-group discriminant analysis was conducted using data collected on active commercial pilots serving with a major air carrier.

\section{Psychological Type}

The Myers-Briggs Type Indicator (MBTI) is a self-reporting, nonjudgmental psychological instrument designed to categorize individuals based on their preferences in four areas: where people obtain their energy (internally or externally), how people perceive their surroundings (denotative or intuitive), their approach to decision making (rational or value oriented), and the approach employed in assessing their environment (judging or perceiving).

Based on Carl Jung's research, Isabel Briggs Myers and Katherine Briggs developed the MBTI instrument, adding an aspect that deals with an individual's lifestyle choices. The self-reporting and self-validating accomplished with the MBTI sorts people into four categories. The first category is extraversion or introversion. The person who indicates a preference for extraversion is one whose energy is directed outward and prefers to interact with people and things. A person who indicates a preference for introversion is one whose energy is directed inward and prefers concepts and ideas. For example, an extrovert might "speak before he or she thinks" and an introvert would probably "think before speaking." The second category is that of perceiving or data collection (sensing or intuition). Those who prefer sensing rely on actual data and pay attention to details. Those who prefer intuition rely on inspiration and look at the "big picture." The third category addresses the decision-making process that people use. Those who prefer thinking make their decision emphasizing logic and principles. Conversely, those who prefer feeling rest their decisions on human values and harmonious relationships. The fourth category addresses lifestyle. In this category people indicate their preferred and most often used mental preference (judging or perceiving). Those who prefer judging indicate decisiveness and task or project completion are important. Those who prefer perception indicate that curiosity and starting a task or project is of higher value. Among active flight officers, over 80 percent fall into two of sixteen categories: ESTJ and ENTJ. There is a dominant category for any generic job classification or profession (Myers \& McCaulley, 1985). Our interest is in the dominant category for successful, professional pilots. Their scoring on the MBTI or another suitable temperament measurement might aid in developing a discriminant function that would serve to screen professional pilot applicants for hire.

\section{Machiavellianism}

Machiavelli's The Prince and The Discourses, in the view of many researchers who study organizational power in administration in both public and private sectors are viewed as viable guides to success. Machiavelli used inductive reasoning and empirical evidence based on his own experiences in formulating his precepts for organizational power. Today the public generally associates the terms power and manipulation with the name of Machiavelli. Christie and Geis (1970) presented Machiavellianism as the concept of interpersonal behavior. A Machiavel is defined as one who is able to influence others to achieve a particular end. To measure Machiavellian orientation, Christie and Geis designed and developed the 
Mach IV and Mach V inventories. According to Christie and Geis (1970), the contrast between a high and low Mach is the degree of freedom from emotional attachment. One with a high Machiavellian orientation:

a. would not be concerned with conventional morality;

b. would conduct one emotionally detached from others with the view that personal involvement would limit the ability of one to treat people as objects;

c. would be concerned primarily with ends rather than means-manipulating others would be a prerequisite for achieving goals; and;

d. would be in full control of faculties, able to assess rationally one's relationship to the psychological environment-neither pathologically disturbed nor possessing a psychosis or neurosis (p.3).

In the formation phase of groups, high Machs tend to emerge as the "key player" or "key man" more so than low Machs; hence, high Machs more frequently guide and direct group planning. This earlier stage of group formation when planning plays a more prominent role, presents a greater opportunity to improvise-a situation tailored to Machiavellian orientation. High Machs exhibit greater detachment from emotions and thus are able to make decisions more effectively and to resist altering opinion after being subjected to counter-argument. Thus, those who score high on the Mach V scale are more likely to be more effective than low Machs in controlling the views of low Machs when conducting group planning activities in initial stages when the environment is less structured.

In attempting to answer the question "how much do high and low Machs exercise manipulations," Christie and Geis (1970) studied people in a laboratory setting where game simulations were conducted. They found that high Machs consistently manipulated more regardless of whether the circumstances were ambiguous or unambiguous. Christie and Geis assert that high Machs are able to assess the weaknesses of people better than low Machs and, thus, are able to capitalize on their weaknesses. This, coupled with a greater insensitivity to people, enables the high Machs to pursue personal or organizational goals more effectively.

How does Machiavellianism relate to cognitive dissonance? Low Machs have difficulties with dissonance traced to higher personal involvement in beliefs whereas high Machs are able to remain detached from personal beliefs and attitudes. High Machs were able to rise above dissonant behavior because of their more practical approach to problem solving. High Machs appear to bargain more effectively in achieving what they want. High Machs appear to be much more aggressive in bargaining, anticipate others to be more aggressive, and are more prone than low Machs to counter aggression with aggression. In addition, high Machs are more inclined to be risk-oriented in their efforts to influence group decision-making. In their assessment of studies of Machiavellianism, Christie and Geis (1970) conclude that those who score higher on the Mach IV and Mach V scales

“. . . manipulate more, win more, and are persuaded less, persuade others more, and otherwise differ significantly from low Machs as predicted in situations in which subjects interact face-to-face with others, when the situation provides latitude for improvisation, and the subject must initiate responses.” (p. 312)

Both experimental and correlational studies suggest that a person's Machiavellian orientation impacts personal behavior-specifically on behavioral patterns in small group settings and relative success in exercising referent power and leadership. (Christie \& Geis, 1970)

\section{METHODOLOGY}

For Group 1 (G1), the population consisted of 52 professional commercial flight officers actively serving on the line. Group 2 was comprised of 40 non-pilot employees of a commercial air carrier. Permission to conduct the survey was obtained from each respondent. The respondents completed the questionnaires in confidence and were guaranteed anonymity regarding the results. Each respondent participating in the study voluntarily submitted data pertaining to MBTI classification. 
Similarly, the respondents completed the Mach $\mathrm{V}$ questionnaires in confidence with anonymity guaranteed; in addition, the author was also able to collect MBTI surveys using the 1998 Keirsey version of the scale.

\section{Instrumentation}

For Groups 1 and 2, quantitative data were collected using the 1998 Keirsey MBTI inventory. The Keirsey instrument is a seventyitem, dyadic, forced-response survey instrument based on the original Myers-Briggs Type Indicator. Professor David Keirsey has investigated personality differences so as to refine his theory of the four temperaments identified in the Myers-Briggs research, and to define the aspects of character that differentiate one from another. His efforts have resulted in his version of the MBTI, The Keirsey Temperament Sorter II, which provides a perspective of how the temperaments differ in the intelligent roles they are likely to develop (Kiersey \& Bates, 1984).

Both the Mach IV and Mach V attitude inventories are derived from the Mach II attitude inventory presented in Likert format to 1,196 college undergraduates in three different universities. Conducting a factor analysis, Christie and Geis selected 20 of the most effective items of the Mach II inventory for further research and analysis. Half of these 20 items were structured so that agreement with them was scored in a positive direction while the other half was reversed so that disagreement with them was scored in a negative direction. The resulting revised 20-item inventory was designated the Mach IV attitude inventory by addressing the possibility of respondents answering in socially desirable way, Christie and Geis (1970) developed the Mach V attitude inventory, a forced response, triadic questionnaire that "makes it difficult for the average respondent to determine which is the socially "correct" answer between the keyed and matched items." (pp. 19-20)

Both the Mach IV and Mach V attitude inventories consist of 20 questions that address the nature of interpersonal tactics, view of human nature, and conventional morality. The Mach IV attitude inventory is a Likert-type questionnaire whose items allow the respondent to answer based upon levels of disagreement or agreement; in contrast, the Mach V contains a force choice pattern that forces the respondent to avoid biasing the selected answer by seeking a socially desirable answer. Contained in each triad of statements is the variable the scale is designed to measure. Included in the triad is another answer similar to the variable statement in social desirability and a third statement that is the antithesis of the other two statements in social desirability. The respondent is directed to pick the statement that is the most accurate in describing personal beliefs and the answer that is the least descriptive of personal beliefs. The Mach V was selected for surveying both Groups 1 and 2 because of the social desirability bias present in the Mach IV instrument.

\section{Data Collection and Statistical Analysis}

Data was collected via a demographic survey, the 1998 Keirsey version of the MBTI and the Mach $\mathrm{V}$ attitude inventory. A discriminant procedure was used to identify a linear combination of quantitative predictor variables that best characterized the differences among the groups. The quantitative predictor variables consisted of the four MBTI dimensions: (1) Extroversion-introversion, (2) Intuiting-Sensing, (3) Thinking-feeling; and (4) Judging-perceiving, and the three Machiavellian variables: (1) conventional morality, (2) interpersonal tactics, and (3) view of people as resources (Huberty, 1984, pp. 156-160).

To derive the discriminant function (Variate), selected first was the method of estimation for assessing a singular variate given two groups. The number of observations or cases classified into the correct group determined the predictive accuracy. A number of criteria were available to determine whether the classification achieved practical or statistical significance. The discriminant function sums the products of the variables multiplied by coefficients. The procedure estimates the coefficients and the resulting function can be used to classify new cases (or, as in the proposed employment of the technique, to identify pilot candidates for hire). The classification of pilot candidates using this function would be based on the temperament and leadership styles of successful professional pilots. 
Simultaneous estimation was employed by computing the Variate so that the predictor variables could be considered concurrently; hence, the Variate was computed based on the entire set of predictor variables regardless of the discriminating power of each predictor variable. This approach was deemed appropriate since the goal was to evaluate each dimension of the complete personality and Machiavellian orientation instruments. The focus on the MBTA and Mach V instruments is based on research that shows successful leaders in informal group settings reflect a specific personality type and Machiavellian orientation different from the general adult population. The average profile of the successful informal group leader would reflect either an ENTJ or ESTJ MBTI category, and a Machiavellian orientation significantly higher than the general adult population norm.

\section{Statistical Significance}

After computing the Variate, the level of significance was assessed by calculating Wilks'
Lambda in order to evaluate the discriminatory power of the Variate. The conventional significance criterion of .05 was used with the view that if the Variate was not significant at or beyond the .05 level, there would be little justification for retaining the Variate. Ninetytwo cases were used in this analysis. By examining the sample means in Figure 1, differences between pilots (Group 1) and nonpilots (Group 2) are noted. The F statistics and significance values in columns three and six are calculated from a one-way ANOVA computed for each variable (see Figure 2). The F statistic equates to the square of the $t$ statistic for a twosample pooled variances $t$ test. Wilks' Lambda indicates differences among groups. The discriminatory value of the MBTI E/I axis appears nil. Based on Wilks' Lambda, the remaining variables are reasonable candidates for inclusion in the discriminant function.

\begin{tabular}{|l|l|l|l|l|}
\hline Group 1 & Mean & Std. & \multicolumn{2}{|l|}{ Valid N (list wise) } \\
\cline { 4 - 5 } Pilots & & Deviation & Unweighted & Weighted \\
\hline Var1 [EI] & 5.1935 & 1.8694 & 52 & 52 \\
Var2 [NS] & 12.451 & 3.1606 & 52 & 52 \\
Var3 [TF] & 13.548 & 3.0314 & 52 & 52 \\
Var4 [JP] & 15.129 & 2.8489 & 52 & 52 \\
Var5 [VIEWS] & 35.612 & 3.7388 & 52 & 52 \\
Var6 [TACTIC] & 40.709 & 3.5795 & 52 & 52 \\
Var7 [CONV]] & 9.1613 & 2.7700 & 52 & 52 \\
\hline Group 2 & Mean & Standard & Valid N (list wise) \\
\cline { 4 - 5 } Non-pilots & & Deviation & Unweighted & Weighted \\
\hline Var1 [EI] & 4.9254 & 1.6173 & 40 & 40 \\
Var2 [NS] & 6.5672 & 2.7819 & 40 & 40 \\
Var3 [TF] & 9.6269 & 2.9120 & 40 & 40 \\
Var4 [JP] & 10.477 & 2.1416 & 40 & 40 \\
Var5[VIEWS] & 38.835 & 2.9418 & 40 & 40 \\
Var6[ ACTIC] & 36.373 & 2.5216 & 40 & 40 \\
Var7 [CONV] & 6.6269 & 2.7015 & 40 & 40 \\
\hline
\end{tabular}

Figure 1. Group Statistics 


\begin{tabular}{|l|l|l|l|l|l|}
\hline & $\begin{array}{c}\text { Wilks' } \\
\text { Lambda }\end{array}$ & $\mathrm{F}$ & \multicolumn{1}{|c|}{ df1 } & df2 & Sig. \\
\hline E-I & .995 & .527 & 1 & 90 & .359 \\
\hline N-S & .525 & 86.964 & 1 & 90 & .000 \\
\hline T-F & .719 & 37.456 & 1 & 90 & .000 \\
\hline J-P & .544 & 80.594 & 1 & 90 & .000 \\
\hline VIEW & .818 & 21.336 & 1 & 90 & .000 \\
\hline TACT & .669 & 47.586 & 1 & 90 & .000 \\
\hline CONV & .839 & 18.359 & 1 & 90 & .000 \\
\hline
\end{tabular}

Figure 2. Tests of Equality of Group Means

\begin{tabular}{|l|l|l|}
\hline \multirow{2}{*}{ Extroversion-Introversion [EI] } & \multicolumn{2}{|c|}{ CATEGORY } \\
\cline { 2 - 3 } & Group One & Group Two \\
\hline Intuiting-Sensing [NS] & 1.579 & 1.162 \\
\hline Thinking-Feeling [TF] & 1.574 & .984 \\
\hline Judging-Perceiving [JP] & .661 & .473 \\
\hline Machiavellian Views [VIEW] & 1.277 & .657 \\
\hline Machiavellian Tactics [TACT] & 2.873 & 3.279 \\
\hline Disregard for Conventional Morality CONV] & .542 & 3.872 \\
\hline (Constant) & -171.486 & .356 \\
\hline
\end{tabular}

Figure 3. Classification Function Coefficients

\begin{tabular}{|c|c|c|c|c|}
\hline Function & Eigenvalue & \% of Variance & Cumulative \% & Canonical Correlation \\
\hline 1 & $2.469 \mathrm{a}$ & 100.0 & 100.0 & .884 \\
\hline
\end{tabular}

Figure 4. Eigenvalue

The classification functions shown in Figure 3 allow the calculation of Fisher's linear discriminant function by taking the difference between the coefficients of the non-pilot and pilot classification functions: $Z=$ (1.5791.162)[EI] + (1.574-.)984[NS] + (0.661-0.473) $[\mathrm{TF}]+(1.277-.657)[\mathrm{JP}]+(2.873-3.279)$ [VIEW] + (4.378 - 3.872) [TACT] + (0.5420.356) [CONV]. Hence, $Z=(0.417)[\mathrm{EI}]+$ $(0.590)[\mathrm{NS}]+(0.188)[\mathrm{TF}]+(0.620)[\mathrm{JP}]-$ $(0.406)[\mathrm{VIEW}]+(0.506)[\mathrm{TACT}]+(0.186)$ [CONV]. The $\mathrm{Z}$ score is the "cut" score for discriminating those applicants matching the desired pilot temperament profile for hire.

The Eigenvalue is the ratio of the betweengroups sum of squares to the within-groups or error sum of squares. The percentage of variance and cumulative percentage of variance are always $100 \%$ for a two-group model such as we have presented. The magnitude of the Eigenvalue indicates strong differentiation between the groups based on the cases used in this study (See Figure 4). If the pilot cases in this study were deemed representative of the cockpit resource management (CRM) standard sought for hire, this specific discriminant function would be useful for current use in pilot selection.

Wilks' lambda is the proportion of the total variance in the discriminant scores not explained by differences between the two groups; in our study, about 29 percent of the variance is not explained by group differences (See Figure 5). We use Wilks' Lambda to test the null 
hypothesis that the means of the variables across the two groups are equal and present little benefit regarding the success of the discriminant function for classifying cases (selecting pilot candidates).

\begin{tabular}{|c|c|c|c|c|}
\hline $\begin{array}{c}\text { Test of } \\
\text { Function(s) }\end{array}$ & $\begin{array}{c}\text { Wilks' } \\
\text { Lambda }\end{array}$ & $\begin{array}{c}\text { Chi- } \\
\text { square }\end{array}$ & df & Sig. \\
\hline 1 & .288 & 115.045 & 7 & .000 \\
\hline
\end{tabular}

Figure 5. Wilks' Lambda

In this study, that null hypothesis is rejected. By transforming Lambda to a variable with a chisquare distribution, we are able to assess whether there is a significant difference between the two group centroids. With a chi-square of 115, a significant difference between the two group centroids (the means of the seven variables calculated simultaneously) is noted.

\begin{tabular}{|l|c|}
\hline Predictor Variables & Function \\
\cline { 2 - 2 } & 1 \\
\hline Extroversion-Introversion [EI] & .212 \\
\hline Intuiting-Sensing [NS] & .513 \\
\hline Thinking-Feeling [TF] & .166 \\
\hline Judging-Perceiving [JP] & .442 \\
\hline Machiavellian Views [VIEW] & -.390 \\
\hline Machiavellian Tactics [TACT] & .438 \\
\hline $\begin{array}{l}\text { Disregard for Conventional } \\
\text { Morality [CONV] }\end{array}$ & .151 \\
\hline
\end{tabular}

Figure 6. Standardized Canonical Discriminant Function Coefficients

Because the predictor variables have different ranges, we have elected to examine the coefficients after they have been standardized. Doing so, allows us to determine those variables having the greatest effect on the model. NS, JP, and TACT appear to discriminate the most in sorting pilot candidates (See Figure 6).

The structure matrix shows the pooled within-groups correlations between discriminating variables and the standardized canonical discriminant function. Variables are ordered by absolute size of correlation within the function (See Figure 7).

Within-group means are computed for each canonical variable, in our study with two categorical groups, the means for our sevenvariable model are -1.259 and 2.270. Figure 8 shows the unstandardized canonical discriminant function evaluated at the group means.

\begin{tabular}{|l|c|}
\hline \multicolumn{1}{|c|}{ Predictor Variables } & Function \\
\cline { 2 - 2 } & 1 \\
\hline Intuiting-Sensing [NS] & .606 \\
\hline Judging-Perceiving [JP] & .583 \\
\hline Machiavellian Tactics [TACT] & .448 \\
\hline Thinking-Feeling [TF] & .398 \\
\hline Machiavellian Views [VIEW] & -.300 \\
\hline $\begin{array}{l}\text { Disregard for Conventional } \\
\text { Morality [CONV] }\end{array}$ & .278 \\
\hline Extroversion-Introversion [EI] & .047 \\
\hline
\end{tabular}

Figure 7. Structure Matrix

\begin{tabular}{|l|c|}
\hline \multicolumn{1}{|c|}{ Category } & Function \\
\cline { 2 - 2 } & 1 \\
\hline Group One & 2.286 \\
\hline Group Two & -1.058 \\
\hline
\end{tabular}

Figure 8 -Functions at Group Centroids

\section{CONCLUSIONS}

The resulting Variate or discriminant function reflects strong discriminant power in identifying those individuals who are successful commercial air carrier pilots from their non-pilot counterparts. Further refinement is needed to include examining the discriminatory power of other instruments suitable for assessing temperament and personality characteristics. There is present a cynicism among many aviation professionals regarding the selection process for pilots and a doubt that new approaches will significantly improve the process in place (Orlady \& Orlady, 1999). Yet the need is present to enhance the means of 'selecting-in' those candidates who match in temperament and attitude the interpersonal aspects of the flight crew environment. 


\section{REFERENCES}

Chidester, T. R., Helmreich, R. L., Gregorich, S. E., \& Geis, C. E., (1991). Pilot personality and crew coordination: Implications for training and selection. The International Journal of Aviation Psychology, 1, 1.

Christie, R., \& Geis, F., (1970). Studies in machiavellianism. New York: Academic Press.

Damos, D., (1996). Pilot selection batteries: Shortcomings and perspectives. The International Journal of Aviation Psychology, 6, 2.

Huberty, C. J. (1984). Issues in the use and interpretations of discriminant analysis. Psychological Bulletin 95, 156-71.

Kiersey, D., \& Bates, M., (1998). Please understand Me II. Del Mar, CA: Prometheus Nemesis Book Company.

Myers, I. B., \& McCaulley, M. H., (1985). Manual: A guide to the development and use of the MyersBriggs type Indicator, Palo Alto, CA: Consulting Psychologists Press.

Orlady, H. W., \& Orlady, L. M., (1999). Human factors in multi-crew flight operations. Aldershot, UK: Ashgate Publishing. 